\title{
Poisson kernels on nilpotent, 3-meta-abelian groups
}

\author{
Richard Penney • Roman Urban
}

Received: 3 April 2014 / Accepted: 29 October 2014 / Published online: 22 November 2014

(C) The Author(s) 2014. This article is published with open access at Springerlink.com

\begin{abstract}
Let $S$ be a semi-direct product $S=N \rtimes A$ where $N$ is a connected and simply connected, nilpotent, non-meta-abelian, 3-abelian Lie group and $A$ is isomorphic with $\mathbb{R}^{k}$, $k>1$. On $S$, we consider a class of second-order left-invariant differential operators of the form $\mathcal{L}_{\alpha}=L^{a}+\Delta_{\alpha}$, where $\alpha \in \mathbb{R}^{k}$, and for each $a \in \mathbb{R}^{k}, L^{a}$ is left-invariant second-order differential operator on $N$ and $\Delta_{\alpha}=\Delta-\langle\alpha, \nabla\rangle$, where $\Delta$ is the usual Laplacian on $\mathbb{R}^{k}$. We prove an upper bound for the Poisson kernel for the operator $\mathcal{L}_{\alpha}$.
\end{abstract}

Keywords Left-invariant differential operators · Poisson kernel · Time-dependent parabolic operators · Brownian motion · Evolution kernel · Diffusion process · 3-Meta-abelian nilpotent Lie groups

Mathematics Subject Classification $\quad 43 \mathrm{~A} 85 \cdot 31 \mathrm{~B} 05 \cdot 22 \mathrm{E} 25 \cdot 22 \mathrm{E} 30 \cdot 60 \mathrm{~J} 25 \cdot 60 \mathrm{~J} 60$

\section{Introduction}

\subsection{Statement of the main result}

Let $S$ be a semi-direct product, $S=N \rtimes A$ where $N$ is a connected, simply connected, 3-meta-abelian, nilpotent Lie group and $A$ is isomorphic with $\mathbb{R}^{k}$. We identify $A$ with its Lie algebra $\mathfrak{a}$. The dimension $k$ of $A$ is called the rank of $S$.

\footnotetext{
R. Penney

Department of Mathematics, Purdue University, 150 N. University St, West Lafayette, IN 47907, USA

e-mail: rcp@math.purdue.edu

R. Urban $(\bowtie)$

Institute of Mathematics, Wroclaw University, Plac Grunwaldzki 2/4,

50-384 Wroclaw, Poland

e-mail: urban@math.uni.wroc.pl
} 
Specifically, we assume that

$$
\begin{aligned}
N & =N_{1} \rtimes\left(N_{2} \rtimes N_{3}\right) \\
& \equiv N_{1} \rtimes N_{2} \rtimes N_{3},
\end{aligned}
$$

where $N_{i}, i=1,2,3$, are abelian Lie groups with the corresponding Lie algebras $\mathfrak{n}_{i}$. To avoid trivialities, we assume that $N_{3}$ is non-central in $N$ (Otherwise, $N$ is meta-abelian.).

Then, there are bases $\left\{X_{1}, \ldots, X_{d_{1}}\right\},\left\{Y_{1}, \ldots, Y_{d_{2}}\right\}$, and $\left\{Z_{1}, \ldots, Z_{d_{3}}\right\}$ for $\mathfrak{n}_{i}, i=1,2,3$, respectively, such that

$$
\left\{X_{i}, \ldots, X_{d_{1}}, Y_{1}, \ldots, Y_{d_{2}}, Z_{1}, \ldots, Z_{d_{3}}\right\}
$$

forms a Jordan-Hölder basis for the Lie algebra $\mathfrak{n}$ of $N$. We assume that these bases are ordered so that the matrix of $\operatorname{ad}_{Z}$ is strictly lower triangular for all $Z \in \mathfrak{n}$. We assume in addition that this basis diagonalizes the $\operatorname{ad}_{\mathfrak{a}}$ action on $\mathfrak{n}$. We use these bases to identify $\mathfrak{n}_{1}$, $\mathfrak{n}_{2}$, and $\mathfrak{n}_{3}$ with $\mathbb{R}^{d_{1}}, \mathbb{R}^{d_{2}}$, and $\mathbb{R}^{d_{3}}$, respectively, and we use the exponential map to identify $N_{i}$ with the corresponding Lie algebras $\mathfrak{n}_{i}=\mathbb{R}^{d_{i}}$.

For $g \in S$, we write $g=x(g) a(g)=x a=(x, a)$, where $x(g)=x \in N$ and $a(g)=a \in$ $A$ denote the components of $g$ in $N \rtimes A$. Similarly, for $x \in N$, we write $x=m(x) v(x) w(x)=$ $m v w=(m, v, w)$, where $m(x)=m \in N_{1}, v(x)=v \in N_{2}$, and $w(x)=w \in N_{3}$ denote the components of $x$ in $N_{1} \rtimes N_{2} \rtimes N_{3}$.

Let

$$
\begin{aligned}
& \Lambda_{1}=\left\{\xi_{1}, \ldots, \xi_{d_{1}}\right\}, \\
& \Lambda_{2}=\left\{\vartheta_{1}, \ldots, \vartheta_{d_{2}}\right\}, \\
& \Lambda_{3}=\left\{\psi_{1}, \ldots, \psi_{d_{3}}\right\},
\end{aligned}
$$

be the roots of the $\operatorname{ad}_{\mathfrak{a}}$ action on $\mathfrak{n}_{1}, \mathfrak{n}_{2}$ and $\mathfrak{n}_{3}$, respectively, corresponding to the given bases. Let

$$
\Lambda=\Lambda_{1} \cup \Lambda_{2} \cup \Lambda_{3}
$$

Hence, for all $H \in \mathfrak{a}$,

$$
\begin{array}{ll}
\operatorname{ad}_{H} X_{i}=\left[H, X_{i}\right]=\xi_{i}(H) X_{i}, & 1 \leq i \leq d_{1}, \\
\operatorname{ad}_{H} Y_{j}=\left[H, Y_{j}\right]=\vartheta_{j}(H) Y_{j}, & 1 \leq j \leq d_{2}, \\
\operatorname{ad}_{H} Z_{k}=\left[H, Z_{k}\right]=\psi_{k}(H) Z_{k}, & 1 \leq k \leq d_{3} .
\end{array}
$$

Let $d=d_{1}+d_{2}+d_{3}$. For $1 \leq i \leq d$, we set

$$
\lambda_{i}= \begin{cases}\xi_{i}, & 1 \leq i \leq d_{1}, \\ \vartheta_{i-d_{1}}, & d_{1}+1 \leq i \leq d_{1}+d_{2}, \\ \psi_{i-d_{1}-d_{2},} & d_{1}+d_{2}+1 \leq i \leq d .\end{cases}
$$

We refer to the class of $N A$ groups defined above as 3-meta-abelian $N A$ groups. Similarly, we can define a class of $k$-meta-abelian $N A$ groups. This is a quite large class of $N A$ groups. It is not difficult to see that the $A N$ parts of classical semisimple Lie groups of type $A_{\ell}, B_{\ell}$,, and $D_{\ell}$ are all $k$-meta-abelian for some $k$.

A simple example of 3-meta-abelian $N A$ group is the group of all upper triangular $4 \times 4$ matrices with positive diagonal. 
The principal object of study in this work is the left-invariant differential operator on $S$,

$$
\mathcal{L}_{\alpha}=\Delta_{\alpha}+\sum_{j=1}^{d_{1}} e^{2 \xi_{j}(a)} X_{j}^{2}+\sum_{j=1}^{d_{2}} e^{2 \vartheta_{j}(a)} Y_{j}^{2}+\sum_{j=1}^{d_{3}} e^{2 \psi_{j}(a)} Z_{j}^{2},
$$

where, for $\alpha=\left(\alpha_{1}, \ldots, \alpha_{k}\right) \in \mathbb{R}^{k}$,

$$
\Delta_{\alpha}=\sum_{i=1}^{k}\left(\partial_{a_{i}}^{2}-2 \alpha_{i} \partial_{a_{i}}\right)
$$

and the $X_{i}, Y_{j}$, and $Z_{k}$ are considered as left-invariant differential operators on $N_{1}, N_{2}$, and $N_{3}$, respectively. We are particularly interested in the bounded harmonic functions for this operator, i.e., bounded functions $F$ on $S$ satisfying $\mathcal{L}_{\alpha} F=0$.

A fundamental result of Damek [3] implies that bounded $\mathcal{L}_{\alpha}$-harmonic functions exist provided the following positivity assumption (which we also assume) holds:

$$
\lambda_{i}(\alpha)>0, \quad \forall i .
$$

In particular, none of the $\lambda_{i}$ are identically 0 and the $\lambda_{i}$ span $\mathfrak{a}^{*}$ (Their joint nullspace consists of vectors annihilated by $\mathrm{ad}_{\mathfrak{a}}$.). We set

$$
A^{+}=\left\{a \in \mathbb{R}^{k}: \lambda_{i}(a)>0 \text { for } 1 \leq i \leq d\right\} .
$$

It also follows from [3] that under our assumptions, the bounded harmonic functions are precisely the "Poisson integrals" of $L^{\infty}(N)$. To describe this concept, let $\chi$ be the modular function for left-invariant Haar measure on $S$. Thus, for all $g \in S$,

$$
\int_{S} f(s g) \mathrm{d} s=\chi(g)^{-1} \int_{S} f(s) \mathrm{d} s
$$

where $\mathrm{d} s$ is left-invariant Haar measure on $S$. Then,

$$
\chi(g)=\operatorname{det}(\operatorname{Ad}(g))=e^{\rho_{0}(a)},
$$

where

$$
\rho_{0}=\sum_{j=1}^{d} \lambda_{j}
$$

Assumption (1.4) together with [3] implies that there exists a Poisson kernel $v$ for $\mathcal{L}_{\alpha}$. That is, there is a $C^{\infty}$ function $v$ on $N$ such that every bounded $\mathcal{L}_{\alpha}$-harmonic function $F$ on $S$ may be written as a Poisson integral against a bounded function $f$ on the quotient space $A \backslash S=N$,

$$
F(g)=\int_{A \backslash S} f(g z) v(z) \mathrm{d} z=\int_{N} f(z) \check{v}^{a}\left(z^{-1} z_{o}\right) \mathrm{d} z, \quad g=\left(z_{o}, a_{o}\right),
$$

where

$$
\check{v}^{a}(z)=v\left(a^{-1} z^{-1} a\right) \chi(a)^{-1}, \text { where } \check{v}(z)=v\left(z^{-1}\right) .
$$

Conversely, the Poisson integral of any $f \in L^{\infty}(N)$ is a bounded $\mathcal{L}_{\alpha}$-harmonic function.

Our goal in this work is to obtain explicit estimates on the rate of decay of $v$ on $N$. To describe our results, we require some additional notation.

For $t \in \mathbb{R}^{+}$and $\alpha \in A^{+}$, let

$$
\delta_{t}^{\alpha}=\left.\operatorname{Ad}((\log t) \alpha)\right|_{N}
$$


Then, $t \mapsto \delta_{t}^{\alpha}$ is a one parameter group of automorphisms of $N$ for which the corresponding eigenvalues on $\mathfrak{n}$ are all positive. It is known $[10,12]$ that then $N$ has $\delta_{t}^{\alpha}$-homogeneous norm: A non-negative and subadditive continuous function $|\cdot|_{\alpha}$ on $N$ which is homogeneous with respect to $\delta_{t}^{\alpha}$, i.e.,

$$
\left|\delta_{t}^{\alpha} x\right|_{\alpha}=t|x|_{\alpha}
$$

and $|n|_{\alpha}=0$ if and only if $n=e$.

For a subset $\Lambda_{o} \subseteq \Lambda$, and $a \in A$, define

$$
\begin{aligned}
\bar{\gamma}_{\Lambda_{o}}(a) & =\min _{\lambda \in \Lambda_{o}} \lambda(a) /\|\lambda\|^{2}, \\
\gamma_{\Lambda_{o}}(a) & =\min _{\lambda \in \Lambda_{o}} \lambda(a) .
\end{aligned}
$$

We set

$$
K(1)=\left\{x \in N:|x|_{\alpha}=1\right\} .
$$

For every $x \in N$, there is precisely one $x_{o} \in K(1)$ such that $\delta_{|x|_{\alpha}}^{\alpha} x_{o}=x$. Thus, we have a map

$$
N \ni x=(m, v, w) \mapsto x_{o}=\left(m_{o}, v_{o}, w_{o}\right) \in K(1) .
$$

The following is our main result.

Theorem 1.1 Let $v$ be the Poisson kernel for the operator $\mathcal{L}_{\alpha}$, defined in (1.3), with $\alpha \in A^{+}$. Under the above assumptions, for every $\varepsilon>0$ there exists a constant $c=c_{\varepsilon, \alpha, \Lambda}>0$ such that for all points $x=(m, v, w) \in N_{1} \rtimes N_{2} \rtimes N_{3}$,

$$
v(x) \leq\left\{\begin{array}{lll}
c\left(1+|x|_{\alpha}\right)^{-\beta_{1}}, & \text { if } & \left|\left(m_{o}, 0,0\right)\right|_{\alpha}>\varepsilon, \\
c\left(1+|x|_{\alpha}\right)^{-\beta_{2}}, & \text { if } & \left|\left(0, v_{o}, 0\right)\right|_{\alpha}>\varepsilon, \\
c\left(1+|x|_{\alpha}\right)^{-\beta_{3}}, & \text { if } & \left|\left(0,0, w_{o}\right)\right|_{\alpha}>\varepsilon,
\end{array}\right.
$$

where

$$
\begin{aligned}
& \beta_{1}=\bar{\gamma}_{\Lambda}(\alpha) \gamma_{\Lambda}(\alpha), \\
& \beta_{2}=\bar{\gamma}_{\Lambda_{2} \cup \Lambda_{3}}(\alpha) \gamma_{\Lambda_{2} \cup \Lambda_{3}}(\alpha), \\
& \beta_{3}=\bar{\gamma}_{\Lambda_{3}}(\alpha) \gamma_{\Lambda_{3}}(\alpha) .
\end{aligned}
$$

In particular, there is a constant $c=c_{\alpha, \Lambda}>0$ such that

$$
\begin{array}{rlrl}
v(m, 0,0) & \leq c\left(1+|(m, 0,0)|_{\alpha}\right)^{-\beta_{1}}, & & \text { for all } m \in N_{1}, \\
v(0, v, 0) \leq c\left(1+|(0, v, 0)|_{\alpha}\right)^{-\beta_{2}}, & & \text { for all } v \in N_{2}, \\
v(0,0, w) \leq c\left(1+|(0,0, w)|_{\alpha}\right)^{-\beta_{3}}, & & \text { for all } w \in N_{3},
\end{array}
$$

Remark 1.2 Notice that $\beta_{1} \leq \beta_{2} \leq \beta_{3}$.

Remark 1.3 We note that [18, Theorem 1.1] says that on a large class of nilpotent Lie groups $N$ containing the 3-meta-abelian groups one has that for every $q>1$, there is $c=c_{q, \alpha, \Lambda}>0$ such that for all $x \in N$,

$$
v(x) \leq c\left(1+|x|_{\alpha}\right)^{-\frac{2}{q} \bar{\gamma}_{\Lambda}(\alpha) \gamma_{\Lambda}(\alpha)} .
$$

In many cases, the estimate in Theorem 1.1 is strictly sharper than (1.12) since clearly the inequalities

$$
\frac{2}{q} \bar{\gamma}_{\Lambda}(\alpha) \gamma_{\Lambda}(\alpha)<\beta_{i} \text { for } i=1,2,3
$$


can hold for many choices of $\Lambda$ and $\alpha$. Also, unlike (1.12), the estimates in Theorem 1.1 differentiate between the various directions of approach to infinity.

Remark 1.4 We should also remark that in [20], we proved the analog of Theorem 1.1 in the 2-meta-abelian case. We found that there are essential difficulties if one wants to prove a similar estimate for the Poisson kernel on $k$-meta-abelian group with $k \geq 3$. In Sect. 1.2 below, we describe these difficulties in more details (See in particular Remarks 1.5 and 1.6.).

\subsection{Strategy of the proof}

Let $\mu_{t}$ (resp., $T_{t}$ ) be the semigroups of measures (resp., operators) generated by $\mathcal{L}_{\alpha}$. It is known (see Sect. 6.1) that the Poisson kernel $v$ is equal to $\lim _{t \rightarrow \infty} \pi_{N}\left(\mu_{t}\right)$, where $\pi_{N}(g)=x(g)$ is a projection from $S$ onto $N$. To get some information on $\mu_{t}$, we use a well-known formula which express $T_{t}$ as a skew-product of the diffusions on $N$ and $A$. (The idea of such a decomposition goes back to [13,14,22]. In the context of $N A$ groups with $\operatorname{dim} A=1$, this decomposition was used in [4-7], and later was generalized by the authors and applied for $\operatorname{dim} A>1$, see, e.g., $[17,19]$.)

Specifically, for $\sigma \in C([0, \infty), A), A=\mathbb{R}^{k}$, let

$$
\mathcal{L}_{N}^{\sigma}=\sum_{j=1}^{d_{1}} e^{2 \xi_{j}(\sigma(t))} X_{j}^{2}+\sum_{j=1}^{d_{2}} e^{2 \vartheta_{j}(\sigma(t))} Y_{j}^{2}+\sum_{j=1}^{d_{3}} e^{2 \psi_{j}(\sigma(t))} Z_{j}^{2},
$$

considered as a time-dependent left-invariant differential operator on $N$. It is known that then $\mathcal{L}_{N}^{\sigma}$ generates a time-inhomogeneous diffusion on $N$ with transition kernel $P_{t, s}^{N_{1} \rtimes N_{2} \rtimes N_{3}, \sigma}$, $t \geq s \geq 0$, and the corresponding evolution operators $U_{s, t}^{N_{1} \rtimes N_{2} \rtimes N_{3}, \sigma}$ (see [2,21]). The skew-product formula says that

$$
T_{t} f(x, a)=\left.\mathbf{E}_{a}^{\sigma} U_{t, 0}^{N_{1} \rtimes N_{2} \rtimes N_{3}, \sigma} f(\cdot, \sigma(t))\right|_{x}=\mathbf{E}_{a}^{\sigma}\left(f *_{N} P_{t, 0}^{N_{1} \rtimes N_{2} \rtimes N_{3}, \sigma}\right)(x, \sigma(t)),
$$

for $f \in C_{c}\left(N \times \mathbb{R}^{k}\right)$ and $t \geq 0$, where the expectation is taken with respect to the distribution of the Brownian motion $\sigma(t) \in \mathbb{R}^{k}$ with drift $-2 \alpha$, starting from $a$, i.e., $\sigma(0)=a$, and generated by $\Delta_{\alpha}$. The subscript $N$ in the convolution $*_{N}$ means that $f$ is convolved with the kernel $P_{t, 0}^{N_{1} \rtimes N_{2} \rtimes N_{3}, \sigma}$ with respect to the first variable in $N$.

The next step is to disintegrate the kernel $P_{t, s}^{N_{1} \rtimes N_{2} \rtimes N_{3}, \sigma}$ using [20, Theorem 1.2] (see also section 3 in [19]) which is a skew-product formula similar to formula (1.14). Specifically, from the decomposition (1.1), the time-dependent family of operators

$$
\mathcal{L}_{N_{2} \rtimes N_{3}}^{\sigma}=\sum_{j=1}^{d_{2}} e^{2 \vartheta_{j}(\sigma(t))} Y_{j}^{2}+\sum_{j=1}^{d_{3}} e^{2 \psi_{j}(\sigma(t))} Z_{j}^{2},
$$

gives rise to an evolution on $N_{2} \rtimes N_{3}=\mathbb{R}^{d_{2}} \rtimes \mathbb{R}^{d_{3}}$ that is described by a kernel $P_{t, s}^{N_{2} \rtimes N_{3}, \sigma}$. The semi-direct product $N_{2} \rtimes N_{3}$ is a 2-meta-abelian group. Thus, we have a relatively good knowledge about the kernel $P_{t, s}^{N_{2} \rtimes N_{3}, \sigma}$ (see [20]). Let $\eta(t)=\left(\eta^{N_{2}}(t), \eta^{N_{3}}(t)\right)$ be the process generated by $\mathcal{L}_{N_{2} \rtimes N_{3}}^{\sigma}$. The skew-product formula from [20] gives that

$$
U_{s, t}^{N_{1} \rtimes N_{2} \rtimes N_{3}, \sigma} f(m, v, w)=\left.\mathbf{E}_{s,(v, w)}^{\eta} U_{s, t}^{N_{1}, \sigma, \eta} f\left(\cdot, \eta^{N_{2}}(t), \eta^{N_{3}}(t)\right)\right|_{m},
$$


where the subscript in the expectation means that $\eta(s)=(v, w)$, and $U_{s, t}^{N_{1}, \sigma, \eta}$ is the family of evolution operators generated by the operator

$$
\mathcal{L}_{N_{1}}^{\sigma, \eta}=\sum_{j=1}^{d_{1}} e^{2 \xi_{j}(\sigma(t))}\left(\left.\operatorname{Ad}(\eta(t))\right|_{\mathfrak{n}_{1}} X_{j}\right)^{2} .
$$

Formula (1.16) allows us to compute and estimate in Sects. 5 and 6 the kernel $P_{t, s}^{N_{1} \rtimes N_{2} \rtimes N_{3}, \sigma}$ on certain subsets of $N$ which, it turns out, are sufficient for our purposes (See formulas (5.1), (6.3), and (6.5).).

Remark 1.5 In [20], we proved a result analogous to Theorem 1.1 for meta-abelian groups, i.e., in the 2-meta-abelian case. The generalization to the 3-meta-abelian case is complicated by the lack of an appropriate estimate for $P_{t, s}^{N_{1} \rtimes N_{2} \rtimes N_{3}, \sigma}$ valid on all of $N$. The fact that it is possible to obtain a good estimate for the Poisson kernel on all of $N$ by piecing together estimates of $P_{t, s}^{N_{1} \rtimes N_{2} \rtimes N_{3}, \sigma}$ on subsets is somewhat surprising.

Remark 1.6 In order to consider $k$-meta-abelian $N A$ groups with $k \geq 4$, we need a better understanding of the situation for $k=3$. In particular, we need to invent some methods which allow us to produce a good and global Gaussian estimate for $P_{t, s}^{N_{1} \rtimes N_{2} \rtimes N_{3}, \sigma}$. If we try to apply methods from [20], the problem of estimating $P_{t, s}^{N_{1} \rtimes N_{2} \rtimes N_{3}, \sigma}$ amounts to the good knowledge of the properties of the time-inhomogeneous process $\eta(t)$ generated by the time-dependent operator (1.15). We hope that if this estimate is obtained, then we will be able to apply induction argument and eventually get estimates for the Poisson kernel for all $k$.

\subsection{Structure of the paper}

The outline of the rest of the paper is as follows. In Sect. 2, we recall some basic facts about exponential functionals of Brownian motion. In Sect. 3, we consider the evolution process $\eta(t)$ on $N_{2} \rtimes N_{3}$ generated by the operator (1.15) and state the estimate for the corresponding transition kernels. Next, in Sect. 4, we study the evolution kernel on $N_{1}$ which is the second ingredient of the skew-product formula (1.16). In Sect. 5, we prove an estimate for the kernel $P_{t, s}^{N_{1} \rtimes N_{2} \rtimes N_{3}, \sigma}$. Finally in Sect. 6, we construct the Poisson kernel and prove our main theorem.

\section{Preliminaries}

\subsection{Exponential functionals of Brownian motion}

Let $b(s), s \geq 0$, be the Brownian motion on $\mathbb{R}$ staring from $a \in \mathbb{R}$ and normalized so that

$$
\mathbf{E}_{a} f(b(s))=\frac{1}{\sqrt{4 \pi s}} \int_{\mathbb{R}} f(x+a) e^{-x^{2} / 4 s} \mathrm{~d} x .
$$

Hence, $b(s)$ has a normal distribution with mean $\mathbf{E} b(s)=a$ and variance $\operatorname{Var} b(s)=2 s$.

Remark Our normalization of the Brownian motion $b(s)$ is different than that typically used by probabilists who tend to assume that $\operatorname{Var} b(s)=s$. 
For $d>0$ and $\mu>0$, we define the following exponential functional

$$
I_{d, \mu}=\int_{0}^{\infty} e^{d(b(s)-\mu s)} \mathrm{d} s .
$$

Theorem 2.1 (Dufresne, [9]) Let $b(0)=0$. Then, the functional $I_{2, \mu}$ is distributed as $\left(4 \gamma_{\mu / 2}\right)^{-1}$, where $\gamma_{\mu / 2}$ denotes a gamma random variable with parameter $\mu / 2$, i.e., $\gamma_{\mu / 2}$ has a density $(1 / \Gamma(\mu / 2)) x^{\frac{\mu}{2}-1} e^{-x} \mathbf{1}_{[0,+\infty)}(x)$.

As a corollary of Theorem 2.1, by scaling the Brownian motion and changing the variable, we get the following theorem (See [17, Lemma 5.4] for details.).

Theorem 2.2 Let $\sigma(u)=b(u)-2 \alpha u$ be the $k$-dimensional Brownian motion with a drift $-2 \alpha, d>0$, and let $\ell \in\left(\mathbb{R}^{k}\right)^{*}$ be such that $\ell(\alpha)>0$. Then,

$$
\mathbf{E}_{a} f\left(\int_{0}^{\infty} e^{d \ell(\sigma(u))} d u\right)=c_{d, \ell, \alpha} e^{\ell(a)} \int_{0}^{\infty} f(u) u^{-\varrho / d} \exp \left(-\frac{e^{d \ell(a)}}{2 d^{2} \ell^{2} u}\right) \frac{d u}{u},
$$

where $\varrho=2 \ell(\alpha) /\|\ell\|^{2}$.

Remark Exponential functionals of type (2.2) are called perpetual functionals in financial mathematics, and they play an important role there (see, e.g., [15,16,23]). In particular, the distribution of the integral over finite interval $(0, t)$ in $(2.2)$ has many applications in Asian options (see, e.g., $[1,8,11]$ ).

2.2 Notation for exponential functionals

For a continuous function $\sigma:[0, \infty) \rightarrow A=\mathbb{R}^{k}$,

$$
\begin{aligned}
& A_{N_{1}, i}^{\sigma}(s, t)=\int_{s}^{t} e^{2 \xi_{i}(\sigma(u))} \mathrm{d} u, \quad i=1, \ldots, d_{1}, \\
& A_{N_{2}, j}^{\sigma}(s, t)=\int_{s}^{t} e^{2 \vartheta_{j}(\sigma(u))} \mathrm{d} u, \quad j=1, \ldots, d_{2}, \\
& A_{N_{3}, k}^{\sigma}(s, t)=\int_{s}^{t} e^{2 \psi_{k}(\sigma(u))} \mathrm{d} u, \quad k=1, \ldots, d_{3},
\end{aligned}
$$

and

$$
A_{N, j}^{\sigma}(s, t)=\int_{s}^{t} e^{2 \lambda_{j}(\sigma(u))} \mathrm{d} u, \quad j=1, \ldots, d .
$$

We also define, for $i=1,2,3$,

$$
A_{N_{i}, \Sigma}^{\sigma}(s, t)=\sum_{j=1}^{d_{i}} A_{N_{i}, j}^{\sigma}(s, t), \quad A_{N_{i}, \Pi}^{\sigma}(s, t)=\prod_{j=1}^{d_{i}} A_{N_{i}, j}^{\sigma}(s, t) .
$$

Finally, we put

$$
A_{N, \Sigma}^{\sigma}(s, t)=\sum_{i=1}^{3} A_{N_{i}, \Sigma}^{\sigma}(s, t), \quad A_{N, \Pi}^{\sigma}(s, t)=\prod_{i=1}^{3} A_{N_{i}, \Pi}^{\sigma}(s, t) .
$$


2.3 Moments of exponential functionals

The following lemma follows from Theorem 2.2.

Lemma 2.3 The functional $A_{N, j}^{\sigma}(0, \infty), j=1, \ldots, d$, has a finite $s$-th moment (for every $a \in \mathbb{R}^{k}$ )

$$
\mathbf{E}^{\sigma} A_{N, j}^{\sigma}(0, \infty)^{s}<+\infty
$$

if and only if $\lambda_{j}(\alpha) /\|\lambda\|^{2}>s$, where $\|\cdot\|$ is $\ell^{2}$ norm on $\mathbb{R}^{k}$. In particular, $A_{N, j}^{\sigma}(0, \infty)$ has all negative moments.

\subsection{Action of $A$ on $N$}

For $a \in \mathbb{R}^{k}, \Phi(a)$ denotes the action of $a$ on $\mathfrak{n}$, see (1.2). By (1.2), the automorphisms $\{\Phi(a)\}_{a \in \mathbb{R}^{k}}$ leave $\mathfrak{n}_{i}, i=1,2,3$ invariant. We identify linear transformation $\Phi(a)$ on $\mathfrak{n}$ with $d \times d$ matrix,

$$
\Phi(a)=\left[\begin{array}{ccc}
\left.\Phi(a)\right|_{\mathfrak{n}_{1}} & 0 & 0 \\
0 & \left.\Phi(a)\right|_{\mathfrak{n}_{2}} & 0 \\
0 & 0 & \left.\Phi(a)\right|_{\mathfrak{n}_{3}}
\end{array}\right],
$$

where

$$
\begin{aligned}
& \left.\Phi(a)\right|_{\mathfrak{n}_{1}}=\operatorname{diag}\left[e^{\xi_{1}(a)}, \ldots, e^{\xi_{d_{1}}(a)}\right], \\
& \left.\Phi(a)\right|_{\mathfrak{n}_{2}}=\operatorname{diag}\left[e^{\vartheta_{1}(a)}, \ldots, e^{\vartheta_{d_{2}}(a)}\right], \\
& \left.\Phi(a)\right|_{\mathfrak{n}_{3}}=\operatorname{diag}\left[e^{\psi_{1}(a)}, \ldots, e^{\psi_{d_{3}}(a)}\right] .
\end{aligned}
$$

Let $\sigma$ be a continuous function from $[0,+\infty)$ to $A=\mathbb{R}^{k}$. We define

$$
\Phi^{\sigma}(t)=\Phi(\sigma(t))
$$

\section{Evolution kernel on $N_{2} \rtimes N_{3}$}

In this section, we consider time-dependent operator on $N_{2} \rtimes N_{3}$,

$$
\mathcal{L}_{N_{2} \rtimes N_{3}}^{\sigma}=\sum_{j=1}^{d_{2}} e^{2 \vartheta_{j}(\sigma(t))} Y_{j}^{2}+\sum_{j=1}^{d_{3}} e^{2 \psi_{j}(\sigma(t))} Z_{j}^{2} .
$$

The operator $\mathcal{L}_{N_{2} \rtimes N_{3}}^{\sigma}$ gives rise to an evolution on $N_{2} \rtimes N_{3}=\mathbb{R}^{d_{2}} \rtimes \mathbb{R}^{d_{3}}$ that is described by a kernel $P_{t, s}^{N_{2} \rtimes N_{3}, \sigma}$ and the corresponding operator $U_{t, s}^{N_{2} \rtimes N_{3}, \sigma}$. The semi-direct product $N_{2} \rtimes N_{3}$ is a 2-meta-abelian group.

The Euclidean space $\mathbb{R}^{n}$ is endowed with the usual scalar product $\langle x, y\rangle=x \cdot y=$ $\sum_{i=1}^{n} x_{i} y_{i}$ and the corresponding $\ell^{2}$ norm $\|x\|=\langle x, x\rangle^{1 / 2}$.

The following estimate is proved in [20, Theorem 4.1]. 
Theorem 3.1 There are positive constants $C, D$ and $k_{o}$ such that for all $t>s \geq 0$ and all $(v, w) \in N_{2} \rtimes N_{3}$,

$$
\begin{aligned}
& A_{N_{2}, \Pi}^{\sigma}(s, t)^{1 / 2} A_{N_{3}, \Pi}^{\sigma}(s, t)^{1 / 2} P_{t, s}^{N_{2} \rtimes N_{3}, \sigma}(v, w) \\
& \leq C\left(\|v\|^{\frac{1}{2 k_{o}}}+1\right) \exp \left(-\frac{D\|w\|^{2}}{A_{N_{3}, \Sigma}^{\sigma}(s, t)}-\frac{D\|v\|^{2}}{\left(\|v\|^{\frac{1}{2 k_{o}}}+\|w\|+2\right)^{2 k_{o}} A_{N_{2}, \Sigma}^{\sigma}(s, t)}\right) \\
& \quad+C A_{N_{3}, \Sigma}^{\sigma}(s, t)^{1 / 2} \exp \left(-D \frac{\|v\|^{\frac{1}{k_{o}}}+\|w\|^{2}}{A_{N_{3}, \Sigma}^{\sigma}(s, t)}\right) .
\end{aligned}
$$

Remark Here, $k_{o}$ is the smallest non-negative integer such that

$$
\left.\left(\operatorname{ad}_{X}\right)^{k_{o}+1}\right|_{\mathfrak{n}_{2}}=0, \quad \forall X \in \mathfrak{n}_{3} .
$$

Note that $k_{o}>0$ since by hypothesis $N_{3}$ is non-central.

\section{Evolution kernel on $N_{1}$}

Let

$$
\eta(t)=\left(\eta^{N_{2}}(t), \eta^{N_{3}}(t)\right)=\left(\eta^{1}(t), \ldots, \eta^{d_{2}+d_{3}}(t)\right)
$$

be the time-inhomogeneous Markov process generated by the operator

$$
\mathcal{L}_{N_{2} \rtimes N_{3}}^{\sigma}=\sum_{j=1}^{d_{2}} e^{2 \vartheta_{j}(\sigma(t))} Y_{j}^{2}+\sum_{j=1}^{d_{3}} e^{2 \psi_{j}(\sigma(t))} Z_{j}^{2}
$$

considered in Sect. 3.

Now on $N_{1}$, we consider time-dependent operator

$$
\mathcal{L}_{N_{1}}^{\sigma, \eta}=\sum_{j=1}^{d_{1}} e^{2 \xi_{j}(\sigma(t))}\left(\left.\operatorname{Ad}(\eta(t))\right|_{\mathfrak{n}_{1}} X_{j}\right)^{2} .
$$

The following notation will be useful. For a $n \times n$ invertible matrix $A$, we set

$$
\mathcal{B}(A)(x)=2^{-1} A^{-1} x \cdot x \text { and } \mathcal{D}(A)=(2 \pi)^{-n / 2}(\operatorname{det} A)^{-1 / 2} .
$$

Since $N_{1}$ is abelian, the transition kernels $P_{t, s}^{N_{1}, \sigma, \eta}$ of the time-inhomogeneous process $\omega(t)$ generated by $\mathcal{L}_{N_{1}}^{\sigma, \eta}$ are given (see Proposition 2.10 in [19]) by

$$
P_{t, s}^{N_{1}, \sigma, \eta}\left(m ; m^{\prime}\right)=\mathcal{D}\left(A_{N_{1}}^{\sigma, \eta}(s, t)\right) e^{-\mathcal{B}\left(A_{N_{1}}^{\sigma, \eta}(s, t)\right)\left(m-m^{\prime}\right)},
$$

where

$$
A_{N_{1}}^{\sigma, \eta}(s, t)=2 \int_{0}^{t}\left[\left.\left.\operatorname{Ad}(\eta(u))\right|_{\mathfrak{n}_{1}} \Phi^{\sigma}(u)\right|_{\mathfrak{n}_{1}}\right]\left[\left.\left.\operatorname{Ad}(\eta(u))\right|_{\mathfrak{n}_{1}} \Phi^{\sigma}(u)\right|_{\mathfrak{n}_{1}}\right]^{*} \mathrm{~d} u,
$$

where $\Phi^{\sigma}(u)$ is defined in (2.3).

Exactly in the same way as Lemma 3.3 in [20], one can prove the following lemma.

Lemma 4.1 There is a constant $C>0$ such that

$$
\mathcal{D}\left(A_{N_{1}}^{\sigma, \eta}(s, t)\right) \leq C A_{N_{1}, \Pi}^{\sigma}(s, t)^{-1 / 2} .
$$




\section{Evolution kernel on $N_{1} \rtimes N_{2} \rtimes N_{3}$}

To get the kernel $P_{t, s}^{N_{1} \rtimes N_{2} \rtimes N_{3}, \sigma}$, we apply the skew-product formula (1.16) (for the proof, see [20, Theorem 1.2]),

$$
P_{t, s}^{N_{1} \rtimes N_{2} \rtimes N_{3}, \sigma}(0,0,0 ; m, v, w)=\lim _{\varepsilon \rightarrow 0} \mathbf{E}_{0,(0,0)}^{\eta} P_{t, s}^{N_{1}, \sigma, \eta}(0 ; m) \psi_{\varepsilon}(\eta(t)),
$$

where for $(v, w) \in N_{2} \rtimes N_{3}=\mathbb{R}^{d_{2}} \rtimes \mathbb{R}^{d_{3}}$ given and $\varepsilon>0$,

$$
\psi_{\varepsilon}\left(v^{\prime}, w^{\prime}\right)=\varepsilon^{-d_{2}-d_{3}} \mathbf{1}_{B_{\varepsilon}(v)}\left(v^{\prime}\right) \mathbf{1}_{B_{\varepsilon}(w)}\left(w^{\prime}\right)
$$

is the (normalized in $L^{1}$ ) indicator function of the product of two $\varepsilon$-balls around $v$ and $w$,

$$
B_{\varepsilon}(v)=\prod_{j=1}^{d_{2}} B_{\varepsilon}^{1}\left(v_{j}\right), \quad B_{\varepsilon}(w)=\prod_{j=1}^{d_{3}} B_{\varepsilon}^{1}\left(w_{j}\right)
$$

and

$$
B_{\varepsilon}^{1}(x)=[x-\varepsilon / 2, x+\varepsilon / 2] .
$$

The following theorem will be used in order to get the estimates for the evolution kernel $P_{t, s}^{N_{1} \rtimes N_{2} \rtimes N_{3}, \sigma}$ on those sets which are necessary for the upper bound for the Poisson kernel.

Theorem 5.1 There is a constant $C>0$ such that for every $t>s \geq 0$, and for every $(m, v, w) \in N_{1} \rtimes N_{2} \rtimes N_{3}$,

$$
P_{t, s}^{N_{1} \rtimes N_{2} \rtimes N_{3}, \sigma}(0,0,0 ; m, v, w) \leq C A_{N_{1}, \Pi}^{\sigma}(s, t)^{-1 / 2} P_{t, s}^{N_{2} \rtimes N_{3}, \sigma}(0,0 ; v, w) .
$$

Proof It follows from (4.2) and Lemma 4.1 that

$$
P_{t, s}^{N_{1}, \sigma, \eta}(0 ; m) \leq P_{t, s}^{N_{1}, \sigma, \eta}(0 ; 0)=\mathcal{D}\left(A_{N_{1}}^{\sigma, \eta}(s, t)\right) \leq C A_{N_{1}, \Pi}^{\sigma}(s, t)^{-1 / 2} .
$$

Then, from (5.1)

$$
P_{t, s}^{N_{1} \rtimes N_{2} \rtimes N_{3}, \sigma}(0,0,0 ; m, v, w) \leq C A_{N_{1}, \Pi}^{\sigma}(s, t)^{-1 / 2} \lim _{\varepsilon \rightarrow 0} \mathbf{E}_{0,(0,0)}^{\eta} \psi_{\varepsilon}(\eta(t))
$$

Clearly,

$$
\lim _{\varepsilon \rightarrow 0} \mathbf{E}_{0,(0,0)}^{\eta} \psi_{\varepsilon}(\eta(t))=P_{t, s}^{N_{2} \rtimes N_{3}, \sigma}(0,0 ; v, w)
$$

and the lemma follows.

\section{Poisson kernel on $N_{1} \rtimes N_{2} \rtimes N_{3}$}

In this section, we will give the proof of Theorem 1.1.

6.1 Construction of the Poisson kernel

Here, we recall the construction of the Poisson kernel $v$ - the principle object of our study. At the same time, this construction provides convenient formula for the Poisson kernel. Let $\mu_{t}$ be the semigroup of probability measures on $S=N \rtimes \mathbb{R}^{k}$ generated by $\mathcal{L}_{\alpha}$. It is known [5] that

$$
\lim _{t \rightarrow \infty}\left(\pi_{N}\left(\check{\mu}_{t}\right), f\right)=(v, f),
$$


where $\pi_{N}$ denotes the projection from $S$ onto $N$ and $(\check{\mu}, f)=(\mu, \check{f}), \check{f}(x)=f\left(x^{-1}\right)$. Let $a \in \mathbb{R}^{k}$ and let $\mu$ be a measure on $N$. We define

$$
\left(\mu^{a}, f\right)=(\mu, f \circ \operatorname{Ad}(a)) .
$$

For $a \in \mathbb{R}^{k}$, we have

$$
v^{a}(x)=v\left(a^{-1} x a\right) \chi(a)^{-1}, \quad x \in N,
$$

where $\chi$ is as in (1.5).

We will need the following fact (see [17, Lemma 4.1] for a proof).

Lemma 6.1 We have

$$
\left(v^{a}, f\right)=\lim _{t \rightarrow \infty}\left(\pi_{N}\left(\check{\mu}_{t}\right)^{a}, f\right)=\lim _{t \rightarrow \infty}\left(\mathbf{E}_{a}^{\sigma} \check{P}_{t, 0}^{N_{3} \rtimes N_{2} \rtimes N_{1}, \sigma}(0,0,0 ; \cdot, \cdot, \cdot), f\right),
$$

where $P_{t, 0}^{N_{3} \rtimes N_{2} \rtimes N_{1}, \sigma}$ is the evolution kernel for the operator $\mathcal{L}_{N}^{\sigma}$ defined in (1.13).

6.2 Upper bound for $v^{s \alpha}$

Our main aim in this subsection is to obtain an upper bound for

$$
v^{s \alpha}(x)=v\left((s \alpha)^{-1} x(s \alpha)\right) \chi(s \alpha)^{-1}
$$

for all $s<0$, where $\alpha \in A^{+}$is a drift vector of the operator (1.3). Then, in Sect. 6.4, in order to get an upper bound for $v$ we will apply a simple homogeneity argument together with some comparison results about evolution kernels.

By Lemma 6.1,

$$
v^{s \alpha}(m, v, w)=\lim _{t \rightarrow \infty} \mathbf{E}_{s \alpha}^{\sigma} \check{P}_{t, 0}^{N_{1} \rtimes N_{2} \rtimes N_{3}, \sigma}(0,0,0 ; m, v, w) .
$$

Hence, in order to estimate $v^{s \alpha}$ we need to estimate $P_{t, 0}^{N_{1} \rtimes N_{2} \rtimes N_{3}, \sigma}$. The main results of this section are the following upper bounds for $v^{s \alpha}$.

Theorem 6.2 Let $\alpha \in A^{+}$, and let $K_{3}$ be a compact subset of $N_{3}$ such that $0 \notin N_{3}$. Then, there is a constant $c=c_{\Lambda, K_{3}, \alpha}>0$ such that for every $s<0$, and all $w \in K_{3} \subset N_{3}$,

$$
v^{s \alpha}(0,0, w) \leq c e^{-\rho_{0}(s \alpha)} e^{s \bar{\gamma}_{\Lambda_{3}}(\alpha) \gamma_{\Lambda_{3}}(\alpha)} .
$$

Theorem 6.3 Let $\alpha \in A^{+}$, and let $K_{2}$ be a compact subset of $N_{2}$ such that $0 \notin N_{2}$. Then, there is a constant $c=c_{\Lambda, K_{2}, \alpha}>0$ such that for every $s<0$, and all $v \in K_{2} \subset N_{2}$,

$$
v^{s \alpha}(0, v, 0) \leq c e^{-\rho_{0}(s \alpha)} e^{s \bar{\gamma}_{\Lambda_{2} \cup \Lambda_{3}}(\alpha) \gamma_{\Lambda_{2} \cup \Lambda_{3}}(\alpha)} .
$$

\subsection{Proofs of Theorems 6.2 and 6.3}

We start with three lemmas. In all of them, the exponential functionals are on the interval $(0, \infty)$, i.e., $A_{\star \star \star}^{\sigma}$ denotes $A_{\star \star \star}^{\sigma}(0, \infty)$.

Lemma 6.4 There is a constant $C>0$ such that for all $s<0$ and $\alpha \in A^{+}$,

$$
\mathbf{E}_{s \alpha}^{\sigma}\left(A_{N, \Pi}^{\sigma}\right)^{-1} \leq C e^{-2 \rho_{0}(s \alpha)}
$$

and

$$
\mathbf{E}_{s \alpha}^{\sigma}\left(A_{N, \Pi}^{\sigma}\right)^{-1} A_{N_{3}, \Sigma}^{\sigma} \leq C e^{-2 \rho_{0}(s \alpha)} .
$$


Proof We have

$$
\mathbf{E}_{s \alpha}^{\sigma}\left(A_{N, \Pi}^{\sigma}\right)^{-1}=e^{-2 \rho_{0}(s \alpha)} \mathbf{E}_{0}^{\sigma}\left(A_{N, \Pi}^{\sigma}\right)^{-1} .
$$

The expected value $\mathbf{E}_{0}^{\sigma}\left(A_{N, \Pi}^{\sigma}\right)^{-1}$ is finite. This follows by applying Cauchy-Schwarz inequality succesively and the fact that exponential functionals $A_{N, j}^{\sigma}$ have negative moments (Lemma 2.3).

Similarly, since $s<0$ and $\alpha \in A^{+}$,

$$
\begin{aligned}
\mathbf{E}_{s \alpha}^{\sigma}\left(A_{N, \Pi}^{\sigma}\right)^{-1} A_{N_{3}, \Sigma}^{\sigma} & =\sum_{j=1}^{d_{3}} \mathbf{E}_{s \alpha}^{\sigma} \prod_{\substack{k=1 \\
k \neq j}}^{d}\left(A_{N, k}^{\sigma}\right)^{-1} \\
& =\sum_{j=1}^{d_{3}} e^{-2 \rho_{0}(s \alpha)+\psi_{j}(s \alpha)} \mathbf{E}_{0}^{\sigma} \prod_{\substack{k=1 \\
k \neq j}}^{d}\left(A_{N, k}^{\sigma}\right)^{-1} \\
& \leq e^{-2 \rho_{0}(s \alpha)} \sum_{j=1}^{d_{3}} \mathbf{E}_{0}^{\sigma} \prod_{\substack{k=1 \\
k \neq j}}^{d}\left(A_{N, k}^{\sigma}\right)^{-1} .
\end{aligned}
$$

Again, by Lemma 2.3, the expectations $\mathbf{E}_{0}^{\sigma} \prod_{\substack{k=1 \\ k \neq j}}^{d}\left(A_{N, k}^{\sigma}\right)^{-1}$ for $j=1, \ldots, d_{3}$ are finite.

The next two lemmas follows immediately from the proof of [17, Lemma 6.2] and the inequality (6.3) on p. 269 in [17].

Lemma 6.5 Let $\alpha \in A^{+}$. For every $\beta>0$, there is a constant $c=c_{\Lambda_{3}, \alpha, \beta}>0$ such that for every $s<0$,

$$
\mathbf{E}_{s \alpha}^{\sigma} e^{-\beta / A_{N_{3}, \Sigma}^{\sigma}} \leq c e^{2 s \bar{\gamma}_{\Lambda_{3}}(\alpha) \gamma_{\Lambda_{3}}(\alpha)} .
$$

Lemma 6.6 Let $\alpha \in A^{+}$. For every $\beta>0$, there is a constant $c=c_{\Lambda_{2}, \Lambda_{3}, \alpha, \beta}>0$ such that for every $s<0$,

$$
\mathbf{E}_{s \alpha}^{\sigma} e^{-\beta /\left(A_{N_{2}, \Sigma}^{\sigma}+A_{N_{3}, \Sigma}^{\sigma}\right)} \leq c e^{2 s \bar{\gamma}_{\Lambda_{2} \cup \Lambda_{3}}(\alpha) \gamma_{\Lambda_{2} \cup \Lambda_{3}}(\alpha)} .
$$

Proof of Theorem 6.2 By Theorem 5.1,

$$
P_{t, 0}^{N_{1} \rtimes N_{2} \rtimes N_{3}, \sigma}(0,0,0 ; 0,0, w) \leq C A_{N_{1}, \Pi}^{\sigma}(0, t)^{-1 / 2} P_{t, 0}^{N_{2} \rtimes N_{3}, \sigma}(0, w) .
$$

From Theorem 3.1, since $0 \notin K_{3}$ there is a constant $c>0$ such that for all $w \in K_{3}$,

$$
\begin{aligned}
& P_{t, 0}^{N_{2} \rtimes N_{3}, \sigma}(0, w) \leq \\
& C A_{N_{2}, \Pi}^{\sigma}(0, t)^{-1 / 2} A_{N_{3}, \Pi}^{\sigma}(0, t)^{-1 / 2}\left(1+A_{N_{3}, \Sigma}^{\sigma}(0, t)^{1 / 2}\right) e^{-c / A_{N_{3}, \Sigma}^{\sigma}(0, t)} .
\end{aligned}
$$

Consequently, for $w \in K_{3}$,

$$
\begin{aligned}
& P_{t, 0}^{N_{1} \rtimes N_{2} \rtimes N_{3}, \sigma}(0,0,0 ; 0,0, w) \\
& \quad \leq C A_{N_{3}, \Pi}^{\sigma}(0, t)^{-1 / 2}\left(1+A_{N_{3}, \Sigma}^{\sigma}(0, t)^{1 / 2}\right) e^{-c / A_{N_{3}, \Sigma}^{\sigma}(0, t)} .
\end{aligned}
$$

Using (6.2), we get that for all $w \in K_{3}$, (note that below the range of integration in all functionals is $(0,+\infty))$

$$
\begin{aligned}
v^{s \alpha}(0,0, w) \leq & C \mathbf{E}_{s \alpha}^{\sigma}\left(A_{N, \Pi}^{\sigma}\right)^{-1 / 2} e^{-c / A_{N_{3}, \Sigma}^{\sigma}} \\
& +C \mathbf{E}_{s \alpha}^{\sigma}\left(A_{N, \Pi}^{\sigma}\right)^{-1 / 2}\left(A_{N_{3}, \Sigma}^{\sigma}\right)^{1 / 2} e^{-c / A_{N_{3}, \Sigma}^{\sigma}} .
\end{aligned}
$$


By the Cauchy-Schwarz inequality

$$
\begin{aligned}
v^{s \alpha}(0,0, w) \leq C & \left(\mathbf{E}_{s \alpha}^{\sigma}\left(A_{N, \Pi}^{\sigma}\right)^{-1}\right)^{1 / 2}\left(\mathbf{E}_{s \alpha}^{\sigma} e^{-2 c / A_{N_{3}, \Sigma}^{\sigma}}\right)^{1 / 2} \\
& +C\left(\mathbf{E}_{s \alpha}^{\sigma}\left(A_{N, \Pi}^{\sigma}\right)^{-1} A_{N_{3}, \Sigma}^{\sigma}\right)^{1 / 2}\left(\mathbf{E}_{s \alpha}^{\sigma} e^{-2 c / A_{N_{3}, \Sigma}^{\sigma}}\right)^{1 / 2}
\end{aligned}
$$

Now Theorem 6.2 follows from Lemma 6.4 and Lemma 6.5.

Proof of Theorem 6.3 By Theorem 5.1,

$$
P_{t, 0}^{N_{1} \rtimes N_{2} \rtimes N_{3}, \sigma}(0,0,0 ; 0, v, 0) \leq C A_{N_{1}, \Pi}^{\sigma}(0, t)^{-1 / 2} P_{t, 0}^{N_{2} \rtimes N_{3}, \sigma}(v, 0) .
$$

From Theorem 3.1 with $w=0$, since $0 \notin K_{2}$ there is a constant $c>0$ such that for all $v \in K_{2}$,

$$
\begin{aligned}
& A_{N_{2}, \Pi}^{\sigma}(0, t)^{1 / 2} A_{N_{3}, \Pi}^{\sigma}(0, t)^{1 / 2} P_{t, 0}^{N_{2} \rtimes N_{3}, \sigma}(v, 0) \\
& \quad \leq C e^{-c_{1} / A_{N_{2}, \Sigma}^{\sigma}(0, t)}+C A_{N_{3}, \Sigma}^{\sigma}(0, t)^{1 / 2} e^{-c_{2} / A_{N_{3}, \Sigma}^{\sigma}(0, t)} \\
& \quad \leq C\left(1+A_{N_{3}, \Sigma}^{\sigma}(0, t)^{1 / 2}\right) e^{-c /\left(A_{N_{2}, \Sigma}^{\sigma}(0, t)+A_{N_{3}, \Sigma}^{\sigma}(0, t)\right)}
\end{aligned}
$$

As in the proof of Theorem 6.2, we conclude that on $K_{2}$, (the range of integration in all functionals below is $(0,+\infty))$

$$
\begin{aligned}
v^{s \alpha}(0, v, 0) \leq & C \mathbf{E}_{s \alpha}^{\sigma}\left(A_{N, \Pi}^{\sigma}\right)^{-1 / 2} e^{-c /\left(A_{N_{2}, \Sigma}^{\sigma}(0, t)+A_{N_{3}, \Sigma}^{\sigma}(0, t)\right)} \\
& +C \mathbf{E}_{s \alpha}^{\sigma}\left(A_{N, \Pi}^{\sigma}\right)^{-1 / 2}\left(A_{N_{3}, \Sigma}^{\sigma}\right)^{1 / 2} e^{-c /\left(A_{N_{2}, \Sigma}^{\sigma}(0, t)+A_{N_{3}, \Sigma}^{\sigma}(0, t)\right)} .
\end{aligned}
$$

This together with Lemma 6.4 and Lemma 6.6 finish the proof.

\subsection{Upper bound for the Poisson kernel $v$}

Having Theorem 5.1, Theorem 6.2, and Theorem 6.3, we are ready to prove the estimate for the Poisson kernel.

Proof of Theorem 1.1 By continuity of $\nu$, there is a constant $C_{\alpha}>0$ such that for all $x \in N$ with the norm $|x|_{\alpha} \leq 1$, we have $v(x) \leq C_{\alpha}$.

Consider $x \in N$ with $|x|_{\alpha}>1$. Let $\delta_{t}^{\alpha}=\operatorname{Ad}((\log t) \alpha)$. Then, $\left|\delta_{t}^{\alpha} x\right|_{\alpha}=t|x|_{\alpha}$. We write $x$ as $x=\delta_{\exp (-s)}^{\alpha} x_{o}$ with $\left|x_{o}\right|_{\alpha}=1$ and $s<0$. Then, $|x|_{\alpha}=e^{-s}>1$. Let $K(1)=\left\{x_{o}\right.$ : $\left.\left|x_{o}\right|_{\alpha}=1\right\}$. By definition (6.1) of $v^{s \alpha}$, we get

$$
v(x)=v\left(\delta_{\exp (-s)}^{\alpha} x_{o}\right)=v\left((s \alpha)^{-1} x_{o}(s \alpha)\right)=e^{\rho_{0}(s \alpha)} v^{s \alpha}\left(x_{o}\right),
$$

where $\rho_{0}=\sum_{j=1}^{d} \lambda_{j}$. Now, estimates (1.10) and (1.11) follow from Theorems 6.3 and 6.2 , respectively, if we apply (6.6) to $x=(0, v, 0)$ and $x=(0,0, w)$, respectively. Then, $x_{o}=\left(0, v_{o}, 0\right)$ and $x_{o}=\left(0,0, w_{o}\right)$. Estimate (1.9) is a consequence of (1.12). Finally, in order to prove (1.8), we proceed as follows.

Let $\mathcal{U}_{t}^{\sigma}(v, w),(v, w) \in N_{2} \rtimes N_{3}$, be the estimate for the evolution kernel $P_{t, 0}^{N_{2} \rtimes N_{3}, \sigma}$ $(0,0 ; v, w)$ given by Theorem 3.1.

By Theorem 5.1,

$$
\begin{aligned}
P_{t, 0}^{N_{1} \rtimes N_{2} \rtimes N_{3}, \sigma}(0,0,0 ; m, v, w) & \leq C A_{N_{1}, \Pi}^{\sigma}(0, t)^{-1 / 2} P_{t, 0}^{N_{2} \rtimes N_{3}, \sigma}(v, w) \\
& \leq C A_{N_{1}, \Pi}^{\sigma}(0, t)^{-1 / 2} \mathcal{U}_{t}^{\sigma}(v, w) .
\end{aligned}
$$


Note that $\check{P}_{t, 0}^{N_{1} \rtimes N_{2} \rtimes N_{3}, \sigma}(0,0,0 ; m, v, w)$ has the same estimate. Now we consider $(m, v, w)$ in a compact sets $K(1)$ (clearly, $0 \notin K(1)$ ). By Lemma 6.1 and (6.7),

$$
\begin{aligned}
v^{s \alpha}(m, v, w)= & \lim _{t \rightarrow \infty} \mathbf{E}_{s \alpha}^{\sigma} \check{P}_{t, 0}^{N_{1} \rtimes N_{2} \rtimes N_{3}, \sigma}(0,0,0 ; m, v, w) \\
& \leq C \mathbf{E}_{s \alpha}^{\sigma} A_{N_{1}, \Pi}^{\sigma}(0, \infty)^{-1 / 2} \mathcal{U}_{\infty}^{\sigma}(v, w) .
\end{aligned}
$$

Notice that it follows from Theorem 3.1 that there is $c>0$ such that for all $(u, w) \in$ $K(1) \cap\left(N_{2} \rtimes N_{3}\right)$, and all $t>0$,

$$
\mathcal{U}_{t}^{\sigma}(v, w) \leq c \mathcal{U}_{t}^{\sigma}(0, w) \text { and } \mathcal{U}_{t}^{\sigma}(v, w) \leq c \mathcal{U}_{t}^{\sigma}(v, 0) .
$$

Applying the above inequalities to (6.8), we get

$$
\begin{aligned}
& v^{s \alpha}(m, v, w) \leq \mathbf{E}_{s \alpha}^{\sigma} A_{N_{1}, \Pi}^{\sigma}(0, \infty)^{-1 / 2} \mathcal{U}_{\infty}^{\sigma}(0, w) \\
& \quad \text { and } v^{s \alpha}(m, v, w) \leq \mathbf{E}_{s \alpha}^{\sigma} A_{N_{1}, \Pi}^{\sigma}(0, \infty)^{-1 / 2} \mathcal{U}_{\infty}^{\sigma}(v, 0) .
\end{aligned}
$$

In fact, the quantities

$$
\mathbf{E}_{s \alpha}^{\sigma} A_{N_{1}, \Pi}^{\sigma}(0, \infty)^{-1 / 2} \mathcal{U}_{\infty}^{\sigma}(0, w) \text { and } A_{N_{1}, \Pi}^{\sigma}(0, \infty)^{-1 / 2} \mathbf{E}_{s \alpha}^{\sigma} \mathcal{U}_{\infty}^{\sigma}(v, 0)
$$

are estimated in the proofs of Theorem 6.2 and Theorem 6.3, respectively. Therefore, we have that for every $\varepsilon>0$, there exists a constant $c=c_{\varepsilon, \Lambda, \alpha}$ such that for all $(m, v, w) \in K(1)$,

$$
v^{s \alpha}(m, v, w) \leq c e^{-\rho_{0}(s \alpha)} e^{s \bar{\gamma}_{\Lambda_{3}}(\alpha) \gamma_{\Lambda_{3}}(\alpha)} \text { if }\|w\| \geq \varepsilon>0
$$

and

$$
v^{s \alpha}(m, v, w) \leq c e^{-\rho_{0}(s \alpha)} e^{s \bar{\gamma}_{\Lambda_{2} \cup \Lambda_{3}}(\alpha) \gamma_{\Lambda_{2} \cup \Lambda_{3}}(\alpha)} \text { if }\|v\| \geq \varepsilon>0 .
$$

Thus by the homogeneity (6.6),

$$
v(m, v, w) \leq c\left(1+|(m, v, w)|_{\alpha}\right)^{-\bar{\gamma}_{\Lambda_{3}}(\alpha) \gamma_{\Lambda_{3}}(\alpha)} \text { if }\left\|w_{o}\right\| \geq \varepsilon>0
$$

and

$$
v(m, v, w) \leq c\left(1+|(m, v, w)|_{\alpha}\right)^{-\bar{\gamma}_{\Lambda_{2} \cup \Lambda_{3}}(\alpha) \gamma_{\Lambda_{2} \cup \Lambda_{3}}(\alpha)} \text { if }\left\|v_{o}\right\| \geq \varepsilon>0 .
$$

The inequality (1.8) follows.

Open Access This article is distributed under the terms of the Creative Commons Attribution License which permits any use, distribution, and reproduction in any medium, provided the original author(s) and the source are credited.

\section{References}

1. Carr, P., Schröder, M.: Bessel processes, the integral of geometric Brownian motion, and Asian options. Teor. Veroyatnost. i Primenen. 48(3), 503-533 (2003)

2. van Casteren, J.A.: Feller semigroups and evolution equations. In: Anastassiou, G.A. (ed.) Series on Concrete and Applicable Mathematics, 12. World Scientific Publishing Co., Pte. Ltd., Hackensack (2011)

3. Damek, E.: Left-invariant degenerate elliptic operators on semidirect extensions of homogeneous groups. Studia Math. 89, 169-196 (1988)

4. Damek, E., Hulanicki, A.: Maximal functions related to subelliptic operators invariant under an action of a solvable Lie group. Studia Math. 101(1), 33-68 (1991)

5. Damek, E., Hulanicki, A.: Boundaries for left-invariant subelliptic operators on semidirect products of nilpotent and abelian groups. J. Reine Angew. Math. 411, 1-38 (1990) 
6. Damek, E., Hulanicki, A., Urban, R.: Martin boundary for homogeneous riemannian manifolds of negative curature at the bottom of the spectrum. Rev. Mat. Iberoam. 17(2), 257-293 (2001)

7. Damek, E., Hulanicki, A., Zienkiewicz, J.: Estimates for the Poisson kernels and their derivatives on rank one NA groups. Studia Math. 126(2), 115-148 (1997)

8. Donati-Martin, C., Ghomrasni, R., Yor, M.: On certain Markov processes attached to exponential functionals of Brownian motion; application to Asian options. Rev. Mat. Iberoam. 17(1), 179-193 (2001)

9. Dufresne, D.: The distribution of a perpetuity, with application to risk theory and pension funding. Scand. Actuarial J. 9, 39-79 (1990)

10. Folland, G.B., Stein, E.: Hardy Spaces on Homogeneous Groups. Princeton University Press, Princeton (1982)

11. Geman, H., Yor, M.: Bessel processes, Asian options, and perpetuities. Math. Financ. 4, 345-375 (1993)

12. Hebisch, W., Sikora, A.: A smooth subadditive homogeneous norm on a homogeneous group. Studia Math. 96(3), 231-236 (1990)

13. Malliavin, P.: Géométrie Différentiale Stochastique, Seminaire de Mathématique Supérieur Été 1997 No 64, Les Press de l'Université de Montréal (1978)

14. Malliavin, M.P., Malliavin, P.: Factorisation et lois limites de la diffusion horizontale au-dessus d'un espace riemannien symétrique. In: Lecture Notes in Math. vol. 404. Springer, Berlin, pp. 164-217 (1974)

15. Matsumoto, H., Yor, M.: Exponential functionals of Brownian motion, I. Probability laws at fixed time. Probab. Surv. 2, 312-347 (2005). (electronic)

16. Matsumoto, H., Yor, M.: Exponential functionals of Brownian motion, II. Some related diffusion processes. Probab. Surv. 2, 348-384 (2005). (electronic)

17. Penney, R., Urban, R.: Estimates for the Poisson kernel on higher rank $N A$ groups. Colloq. Math. 118(1), 259-281 (2010)

18. Penney, R., Urban, R.: An upper bound for the Poisson kernel on higher rank $N A$ groups. Potential Anal. 35(4), 373-386 (2011)

19. Penney, R., Urban, R.: Estimates for the Poisson kernel and the evolution kernel on the Heisenberg group. J. Evol. Equ. 12(2), 327-351 (2012)

20. Penney, R., Urban, R.: The evolution and Poisson kernels on nilpotent meta-abelian groups. Studia Math. 219(1), 69-96 (2013)

21. Stroock, D.W., Varadhan, S.R.S.: Multidimensional diffusion processes. Reprint of the 1997 edition. Classics in Mathematics. Springer, Berlin (2006)

22. Taylor, J.C.: Skew products, regular conditional probabilities and stochastic differential equations: a technical remark in Séminaire de Probabilité XXVI, Lecture Notes in Math. 1526. Springer, Berlin, pp. 299-314 (1992)

23. Yor, M.: Exponential functionals of Brownian motion and related processes. With an introductory chapter by Hélyette Geman. Chapters 1, 3, 4, 8 translated from the French by Stephen S. Wilson. Springer Finance. Springer, Berlin (2001) 